\title{
Magnetic dissipation force microscopy studies of magnetic materials (invited)
}

\author{
Y. Liu and P. Grütter ${ }^{\mathrm{a})}$ \\ Centre for the Physics of Materials, Department of Physics, McGill University, Montréal, Québec, \\ H3A 2 T8 Canada
}

\begin{abstract}
We describe the principles of dissipation measurements, discuss various eddy current damping mechanisms, give a brief review of a model for magnetoelastic dissipation due to domain-wall width oscillations, and present some applications of magnetic dissipation force microscopy to magnetic materials. Energy dissipation is measured by simultaneous monitoring of the damping of an oscillating cantilever and the shift in resonant frequency in a magnetic force microscope. Magnetoelastic dissipation is caused by tip-field-induced domain-wall width oscillations through magnetostriction effects. Magnetoelastic damping is strongly correlated with micromagnetic structures and allows different domain walls (such as Bloch and Néel walls) to be distinguished.

(C) 1998 American Institute of Physics. [S0021-8979(98)48611-0]
\end{abstract}

Magnetic dissipation force microscopy ${ }^{1-4}$ is a working mode of an ac magnetic force microscope (MFM). In this mode, the magnetic tip oscillating at resonance serves as both force gradient and dissipation sensor. Energy dissipation causes damping of the oscillating tip and is measured by monitoring the oscillation amplitude.

This new working mode of the ac MFM directly measures the local magnetic energy dissipation with lateral resolution at least as good as the normal MFM. We do this by measuring the damping of the oscillating tip in a MFM simultaneously with the usual frequency shifts associated with tip-sample force gradient variations. A change in damping of the MFM probe is the result of energy transferred between the tip and the sample and is detected as a difference in cantilever oscillation amplitude. Damping due to Joule dissipation in semiconductors has previously been measured with a different detection scheme by Denk and Pohl. ${ }^{5}$

In the ac MFM, a magnetic tip is vibrated above a sample surface and creates a local alternating magnetic field at the sample, resulting in energy dissipation in the sample. The thin-film tip stray field, which contains a dc part and an ac part, is concentrated on a region of the sample $50-500 \mathrm{~nm}$ in diameter, depending on the tip shape and tip-sample separation. ${ }^{2}$ By using this highly localized tip field and measuring the resulting energy dissipation, we can quantitatively determine the local effect of the tip field on the micromagnetic structure of the sample.

We measure the damping $\gamma$ or equivalent quality factor $Q$ change of the cantilever, which can be described by a driven damped harmonic oscillator $\left(\gamma=k_{l} / \omega_{0} Q\right)$. Here, $k_{l}$ is the cantilever spring constant and $\omega_{0}$ is the resonant frequency of the cantilever. At resonance, the vibration amplitude is $A=\left(F_{0} / \omega_{0}\right) Q_{0}$, where $F_{0}$ is the driving amplitude

\footnotetext{
${ }^{a)}$ Electronic mail address: grutter@physics.mcgill.ca
}

(proportional to the ac voltage applied to the piezoelectric bimorph) and $Q_{0}$ is the quality factor of the cantilever. Magnetic dissipation in the sample causes an energy loss in the cantilever and so reduces the $Q$ factor. A larger driving force is thus needed to keep the vibration amplitude constant. The extra force is given by

$$
\Delta F=-\left(F_{0} / Q_{0}\right) \Delta Q .
$$

The energy loss (dissipation) in one cycle is then

$$
\Delta E=-\left(E_{k} / Q_{0}^{2}\right) \Delta Q .
$$

Here, $E_{k}=(1 / 2) k_{l} A^{2}$ is the vibration energy of the cantilever with $k_{l}$ the spring constant of the cantilever.

We can thus measure the energy loss (dissipation) by measuring the driving force change while keeping the cantilever vibrating at resonance with a constant amplitude if we ensure that any phase shifts are negligible. By recording the driving signal changes simultaneously with the usual frequency shifts associated with tip-sample force gradient variations, a normal MFM image and a dissipation image can be acquired simultaneously. This allows the study of correlations between magnetic dissipation and domain structure. In the following, we will use $\Delta Q$ in our discussion. $\Delta Q$ can easily be transformed to $\Delta E$ or $\Delta \gamma$.

We employ the same phase-locked loop (PLL) demodulator ${ }^{6}$ to track both the resonant frequency and the driving force of the cantilever. When the PLL is locked to the cantilever resonance, it will always keep the cantilever vibrating on resonance and at a constant vibration amplitude by adjusting the driving amplitude to compensate for any damping. This is achieved with an additional feedback circuit to keep the cantilever's vibration amplitude constant at a preset value. A dc voltage proportional to the cantilever's vibration amplitude is compared with a set value and the difference signal is integrated. The output signal of the inte- 
grator will determine the driving signal amplitude to a piezoelectric bimorph, which excites the cantilever. The integrator output serves as the signal for dissipation images.

The fundamental limit for the detection of a $Q$-factor change $\delta Q$, assuming that the displacement measurement is limited only by the cantilever's thermal motion, is given by

$$
\delta Q_{\text {thermal }}=\frac{1}{A} \sqrt{\frac{2 k_{B} T Q^{3} B_{W}}{k_{l} \omega_{0}}},
$$

with $k_{B}$ being the Boltzmann's constant, $T$ being the temperature, and $B_{W}$ being the measurement bandwidth. For our cantilevers $\left(k_{l}=0.1 \mathrm{~N} / \mathrm{m}, f_{0}=30 \mathrm{kHz}\right.$, and $Q=1000$ in vacuum) $\delta Q_{\text {thermal }}=5.3$ (which corresponds to a $\delta \gamma_{\text {thermal }}$ $=2.8 \mathrm{pNs} / \mathrm{m}$ or $\Delta E=1.0 \times 10^{-3} \mathrm{eV}$, respectively) with $B_{W}$ $=35 \mathrm{~Hz}$ and $A=25 \mathrm{~nm}$. All our dissipation data are thermally limited. A better signal-to-noise ratio is predicted, and indeed, observed by measuring dissipation in vacuum. All of the data presented here were obtained at $5 \times 10^{-5}$ mbar.

Minimizing the phase error between the cantilever oscillation and its drive signal is crucial to obtaining a meaningful damping measurement. Phase errors (e.g., due to filters) will drive the cantilever off-resonance. A larger drive amplitude $F^{\prime}$ would then be necessary to maintain a constant amplitude, which would falsely be interpreted as a change in cantilever damping. The $Q$-factor error $\delta Q$, due to phase error $\delta \phi$, is given by

$$
\delta Q_{\text {Phase }} \approx \frac{1}{2} Q(\delta \phi)^{2} .
$$

Our carefully optimized electronics maintain a phase error of less than $0.003 \mathrm{rad}$ for frequencies between $10 \mathrm{kHz}$ and $2 \mathrm{MHz}$. This amounts to an error of $\delta Q<0.005$ for $Q_{0}$ $=1000$, substantially smaller than the thermal limit. A second source of phase shifts is frequency feedback errors, e.g., as a result of varying force gradients between tip and sample. The influence of frequency error on the drive output can directly be measured by modulating the PLL reference frequency. We determined $\delta Q<0.004$ for a $10 \mathrm{~Hz}$ modulation in vacuum. In our experiments, the frequency feedback errors are kept smaller than $2 \mathrm{~Hz}$, resulting in $\delta Q<0.001$. A further potential phase error is particular to our fiber-optic interferometric deflection sensing technique. Dc deflection $\Delta z$ as a result of forces acting on the cantilever will result in an optical path-length difference, and thus, a phase-shift $\Delta \phi=(2 \Delta z / \lambda) 2 \pi$. In our experiments we monitor this deflection simultaneously with the damping and the force gradient signal. The maximum total deflection is always smaller than $1 \mathrm{~nm}$, and thus, introduces a phase error of 0.01 , resulting in an error of $\delta Q<0.05$.

A further potential error in damping measurements is due to the tip-sample separation dependence of the hydrodynamic cantilever damping observed in air. ${ }^{7}$ Typically, $Q$ changes larger than the thermal limit are observed for tipsample separation changes of a few $\mathrm{nm}$ if the average separation is $50 \mathrm{~nm}$ or less. To avoid the potential convolution of the regular MFM (acquired at variable tip-sample reparation as $F^{\prime}=$ const.) and dissipation data, we always acquire our dissipation data in vacuum, where a separation-dependent $Q$ factor is not observed for tip-sample separations larger than several $\mathrm{nm}$.
In conclusion, by carefully designing and characterizing our PLL, keeping feedback errors to $2 \mathrm{~Hz}$, and controlling phase errors due to optical path-length changes and vacuum operation, we are confident that all measured drive amplitude changes are only due to changes in the magnetic damping of the cantilever.

The following loss mechanisms due to eddy and induced currents can be identified:

(1) Eddy currents in the sample due to oscillations of the domain-wall position. An ac magnetic field may lead to domain-wall oscillations around the wall equilibrium position, generating eddy currents around the oscillating domain walls.

(2) Eddy currents in the MFM tip due to the stray field from the sample. When the tip is oscillating, the sample stray field generates eddy currents in the tip.

(3) Eddy currents in the tip due to tip magnetization changes. When the tip is oscillating, the gradient of the sample stray field may induce tip magnetization change $d M / d t$, which generates eddy currents in the tip.

(4) Eddy currents in the sample due to tip field. An oscillating tip creates an alternating magnetic field at the sample, thus leading to magnetic-flux changes in the sample. For a conducting sample, eddy currents are induced in the sample.

(5) Eddy currents in the sample due to domain-wall jumps.

(6) Induced currents in the sample due to capacitance changes between the tip and sample. The capacitance of the tip-sample system oscillates as a result of the tip oscillation. If a constant voltage is applied between the tip and the sample (as in our instrument for servopurpose ${ }^{8}$ ), the oscillating capacitance leads to an alternating current as a result of alternating charge redistribution. Variations in magnetoresistance could thus lead to magnetic contrast. This source of damping is not related to eddy currents.

We have theoretically calculated and experimentally tested the contributions of eddy current losses (1), (2), and (3) to the damping of a $\mathrm{Si}$ cantilever (coated with $20 \mathrm{~nm}$ CoNi film) and a $\mathrm{Si}_{3} \mathrm{~N}_{4}$ cantilever (coated with $90 \mathrm{~nm} \mathrm{CoP-}$ tCr film) on several samples (30 nm thick pattered $\mathrm{Ni}_{80} \mathrm{Fe}_{20}$ film, $4 \mathrm{~nm}$ thick Co film, and $40 \mathrm{~nm}$ thick CoPtCr film) and found that these contributions are far too small (by 4-6 orders of magnitude) to explain the observed damping signal. $^{2,3}$ The damping signals due to losses (4) and (6) depend on the resistivity of the sample. The dissipation due to induced currents (6) is proportional to the sample's resistivity and can be used to determine dopant concentration in semiconductors. ${ }^{5}$ The resistivity of the metallic samples is low (for cobalt it is $9.8 \times 10^{-8} \Omega \mathrm{m}$ ), which leads to a dissipation signal that is below the thermal limited minimum detectable resistivity change of about $4 \times 10^{-3} \Omega \mathrm{m} .{ }^{5}$ The small variations of resistivity due to magnetoresistive effects (typically, $\Delta R / R<1 \%$ ) do not lead to a measurable dissipation contrast. The dissipation due to eddy current (4) is an inverse measure of resistivity. However, a dissipation measurement on a patterned permalloy film on a $\mathrm{Si}$ substrate ${ }^{1}$ shows that the background dissipation signal on the permal- 
loy area (with a resistivity on the order of $10^{-7} \Omega \mathrm{m}$ ) is smaller than in the $\mathrm{Si}$ area (with a much higher resistivity). The permalloy background signal corresponds to the thermal limit, in contrast to the observed resistive dissipation on $\mathrm{Si}$. We conclude that the effect of the induced current (6) is larger than that of the eddy current (4) in this sample. Due to the very short time scales involved in domain-wall jumps, eddy currents (5) might lead to some dissipation contrast. ${ }^{3}$ In wall jumps (induced by the tip field), the wall speed can be extremely high, resulting in substantial, but difficult to quantify, eddy current losses.

In conclusion, all eddy current related processes, with the exception of tip-induced domain-wall jumps, lead to dissipation values not measurable in vacuum with our standard MFM tips.

A magnetoelastic model for dissipation, which bases the dissipation on domain-wall width oscillations and the resulting phonon emission through the magnetostriction effect, has given quantitative agreement with experiments on samples investigated in detail. ${ }^{2,4}$ The domain-wall width oscillations $W(t)$ can be treated as a simple harmonic oscillator described by an equation, which contains an inertia term $m \ddot{W}$, a stiffness force $\alpha(\Delta W)$, a damping term, ${ }^{9}$ and an external alternating driving force term related to the external ac tip field. Here, $m$ is the effective mass of the domain wall for width oscillations and $\alpha$ is the wall stiffness of the domain wall for width oscillations. In most problems, damping terms are considered to be viscous in nature (i.e., the damping force is proportional to velocity). However, for magnetoelastic loss, the damping of wall width oscillations appears to be frictional in nature since the elastic energy changes associated with the wall width change only depend on the magnitude of the wall width change and not on the rate of the width change. Hence, the damping term can be written as $-\beta \dot{W} /|\dot{W}|$. The equation of motion for wall width oscillations is then

$$
m \ddot{W}+\beta \frac{\dot{W}}{|\dot{W}|}+\alpha\left(W-W_{0}\right)=F,
$$

where $W_{0}$ is the equilibrium width of the domain wall and $F=F_{0} \exp (i 2 \pi f t)$ is the force trying to oscillate the wall width.

The parameters $m, \alpha, \beta$, and $W_{0}$ have been derived and expressed in basic magnetic properties of magnetic materials $^{4}$ with $m=\pi \mu_{0} / 48 \gamma^{2} W_{0}, \quad \alpha=K_{1} / W_{0}, \quad \beta=\frac{1}{2} c \lambda^{2}$, and $W_{0}=\sqrt{2 J S^{2} \pi^{2} / K_{1} a}$, assuming that the demagnetization energy of the wall can be neglected. Here, $K_{1}$ is the anisotropy constant; $c$ is Young's modulus; $\lambda$ is the magnetostriction constant; $J$ is the exchange integral; $S$ is the spin; $a$ is the unit cell length; and $\gamma$ is the gyromagnetic ratio. The driving force for wall width oscillations $F$ was found to be strongly dependent on the relative orientation of the meanmagnetization direction inside a domain wall and the external magnetic field. For a Bloch wall $F= \pm\left(2 \mu_{0} M_{s} H_{z} / \pi\right)$, depending only on the out-of-plane component of the external tip field, while for a Néel wall $F$ is related to the in-plane component of the external tip field. In magnetic dissipation force microscopy, the external field is created by an oscillat- ing magnetic tip and is highly localized. Thus, different micromagnetic structures in a domain wall will result in dissipation contrast for a given tip. Letting $F=F_{0} \exp (i 2 \pi f t)$ and $W=W_{0}+W_{1} \exp [i(2 \pi f t+\delta)](f$ is the oscillation frequency of the tip), we can obtain the energy dissipation in one oscillation cycle as

$$
P=4 f \beta W_{1}=4 f \beta \frac{\sqrt{F_{0}^{2}-\beta^{2}}}{\alpha-(2 \pi f)^{2} m} .
$$

Quantitative agreement between this equation and experiments for a Co film sample and a $\mathrm{Co} / \mathrm{Ni}$ multilayer sample was previously obtained. ${ }^{2,4}$ Furthermore, by comparing permalloy and Terfenol-D (which has a much higher $\lambda$ than permalloy), we observed the expected relative dissipation increase in the Terfenol-D sample. In addition to this agreement, the model predicts a wall width resonance. The resonant frequency for the Co sample was calculated to be on the order of $10^{10} \mathrm{~Hz} .{ }^{4}$ Hence, the wall width resonance might influence the high-frequency properties of magnetic materials.

An interesting consequence of Eq. (6) is that a minimum driving force is necessary to observe dissipation. By engineering suitable tips (e.g., by selecting a thin, low moment coating), a small $F_{0}$ can be created, thus inhibiting energy loss due to domain-wall width oscillations. The absence or presence of nonconservative tip-sample interactions (at least above the thermal limit) can thus be monitored by dissipation force microscopy.

Magnetic dissipation force microscopy has been used to study the magnetic domain structures of several samples, including a patterned permalloy film, ${ }^{1,4}$ and transitions in a $\mathrm{CoPtCr}$ magnetic recording medium, ${ }^{1} \mathrm{Co} / \mathrm{Ni}$ multilayer, ${ }^{2}$ and Terfenol-D. ${ }^{1,4}$ The experimental dissipation showed discontinuous change along domain walls in the patterned permalloy film. ${ }^{1}$ This suggests that there are different micromagnetic structures in the domain walls. Figure 1 shows a highresolution MFM image and the simultaneously acquired magnetic dissipation image on a part of one permalloy square. It can be seen from Fig. 1(a) that the magnetic domain walls in this permalloy are not pure Néel or pure Bloch walls. They contain complicated micromagnetic structures, possibly Bloch points and Bloch lines. We observe that on the same sample but for different squares, domain walls have different micromagnetic structures. In Fig. 1(b) the long micromagnetic features (indicated by the arrows), which cross the main wall and connect the two adjacent magnetic domains, are formed by finer identical spots. Black and white spots are observed. They show opposite signs of magnetic tip-sample interactions. Other domain walls even on the same square show less magnetic substructure [top left of Fig. $1(\mathrm{a})]$.

Figure 2(a) gives another example of a domain-wall structure observed on the permalloy film. Here, there are fewer internal micromagnetic features inside the main domain wall, which shows up as a bold dark line from the lower-left corner to the upper-right corner (the bold white line on the left side of the image is the edge of the permalloy square). However, one observes a lot of ripplelike magnetic features, which are perpendicular to and terminated at the 

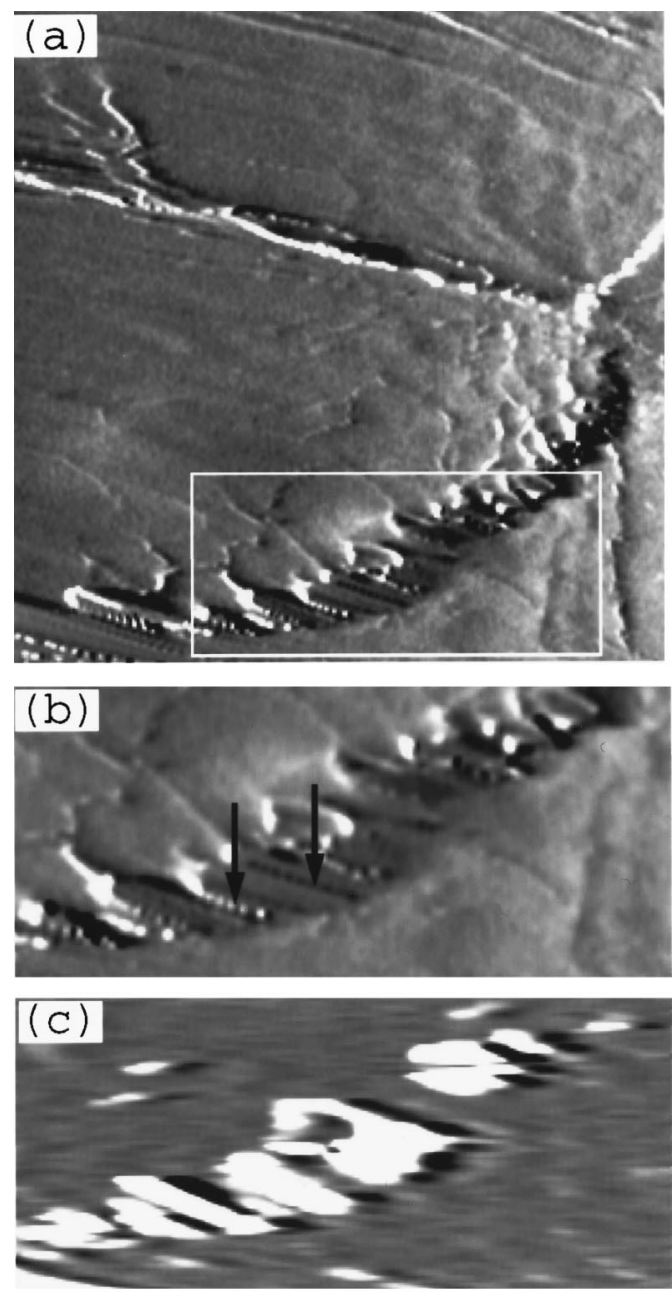

FIG. 1. High-resolution MFM and magnetic dissipation images on a permalloy square with a $20 \mathrm{~nm}$ CoNi film coated Si tip. The MFM image is differentiated along the fast scan direction to enhance the contrast. (a) is the $12 \mu \mathrm{m} \times 12 \mu \mathrm{m}$ MFM image, (b) is the enlarged area shown by the rectangle in (a), and (c) is the magnetic dissipation image of the same area as (b)

main wall position. Ripples in permalloy films are commonly observed ${ }^{10}$ and have been observed by MFM. ${ }^{11}$ Figure 2(b) is the simultaneously acquired dissipation image, which shows again that the dissipation signal is associated with some of the magnetic wall features. There is a magnetic feature (indicated by an arrow), which leads to a pronounced dissipation signal (about $0.03 \mathrm{eV}$ in one oscillation cycle), and is not part of the main wall. A reproducible jump of this magnetic feature is observed as a highly localized change in dissipation on several scan lines indicated by an arrow in Fig. 2(b). By applying a field of only 2 Oe with an in situ electromagnet, this feature disappears while the main wall is displaced by less than $1 \mu \mathrm{m}$, confirming that the feature is magnetic in origin.

The existence of different micromagnetic domain-wall structures of the permalloy film suggest that there are many wall configurations which have very similar energy minima. In order to advance our understanding of the micromagnetic wall structures and the associated magnetic dissipation in patterned permalloy films, micromagnetic calculations are needed. Such calculations are not presently feasible for sys-
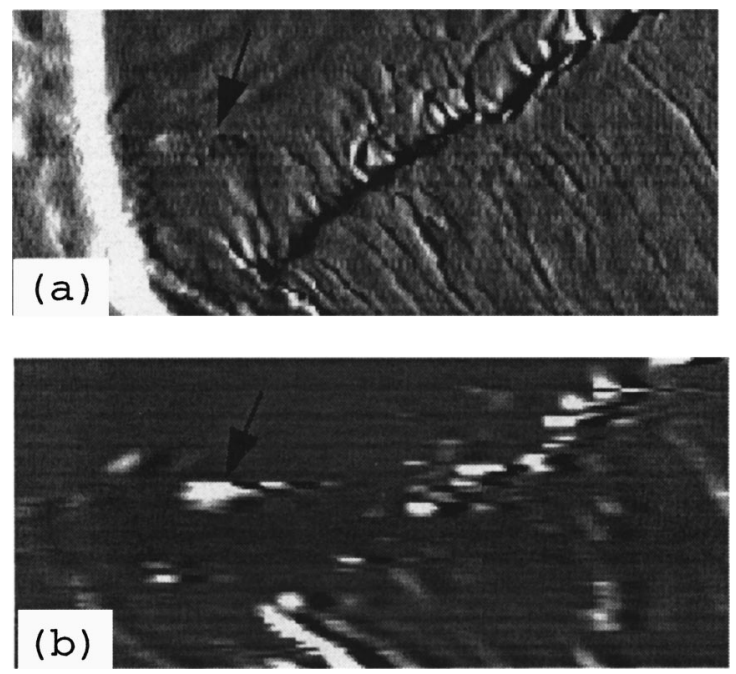

FIG. 2. Ripplelike magnetic features intersect with this main wall in a permalloy film. The image area is $8 \mu \mathrm{m} \times 4 \mu \mathrm{m}$. (a) differentiated MFM image. The domain wall is the black structure running diagonally across the image. The white vertical line to the left of the image is the edge of the permalloy square. (b) Magnetic dissipation image.

tems as large as the $20 \mu \mathrm{m}$ squares. Clearly, however, dissipation imaging opens the possibility of characterizing a variety of micromagnetic structures.

For this study, we used our home-built high-resolution magnetic dissipation force microscope (MDM) with an in situ magnetizing stage. The magnetizing stage is also home-built ${ }^{12}$ and the field (in the sample plane) can be changed continuously from -1 to $+1 \mathrm{kOe}$. The MFM and MDM images are taken simultaneously as a function of this external magnetic field. Prior to experiments, the sample is saturated and then ac demagnetized. The procedure for changing the field follows the macroscopic $M-H$ hysteresis loop. We first increase the field from zero to saturation and then decrease it to zero. An opposite field is then applied to the opposite saturation value and decreased to zero again. The procedure is then finished by again changing the sign of the field and increasing it to the first saturation value. The field is changed in small steps. After each of these steps, a MFM and a MDM image are taken simultaneously. For each experimental run, typically, 20-30 images are taken and assembled as a video to allow easier observation of changes between field steps.

Figure 3 shows the evolution of the domain configuration with decreasing magnetic field after saturating along one edge of the permalloy square. Figure 4 gives the simultaneously acquired dissipation images. The image of the permalloy is not square due to uncorrected piezo-nonlinearity.

The saturation field is $90 \mathrm{Oe}$ for the patterned permalloy film, at which no contrast is observed in either MFM or dissipation images. This saturation field here is much higher than for a film of the same thickness, but infinite extent in the plane of the film (which has a coercivity of around $2 \mathrm{Oe}$ ). The higher saturation field in the patterned film is due to demagnetization effects, since for an external field larger than 10 Oe the domain walls are concentrated at the edge area of the squares. A much higher external field is needed to 

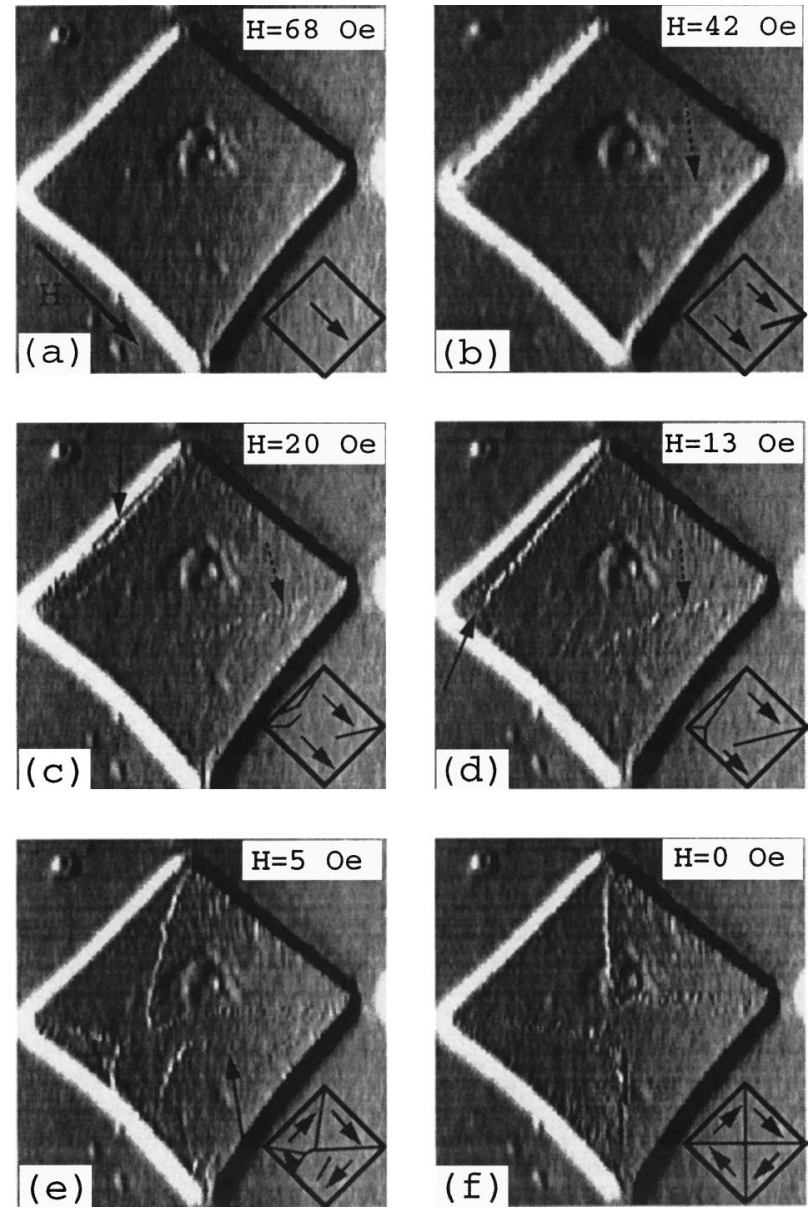

FIG. 3. Evolution of the domain configuration with decreasing magnetic field after saturating along one edge of the permalloy square. The field direction is indicated in (a). (a)-(f) are taken consecutively with the in situ applied field shown in the individual images. The inset in each individual image depicts the proposed domain structure.

remove these domain walls. On decreasing the field, magnetization reversal takes place first by reverse-domain nucleation at one edge at a field of 68 Oe [Figs. 3(a) and 4(a)]. Note that although nucleation is not seen in the regular MFM image, it is clearly seen in the dissipation image. Hence, dissipation imaging allows the observation of early stage domain nucleation especially at sample edges, where other magnetic imaging techniques have major problems. On decreasing the field further, the reverse domain grows [as indicated by a solid arrow in Fig. 3(c)] with its magnetization direction probably perpendicular to the external field (parallel to the edge). At $H=13 \mathrm{Oe}$, a new domain with a magnetization direction antiparallel to the external field (parallel to one edge of the sample) is formed. Figures 3(b), 3(c), and 3(d) also show a long magnetic structure (indicated by a dashed arrow) starting from the right corner of the square. This structure, becoming longer with decreasing external field, is believed to be a $360^{\circ}$ wall (simulations of the expected MFM response of a $360^{\circ}$ wall are comparable with experimental results). ${ }^{3}$ At $H=5 \mathrm{Oe}$, all the four domains with parallel, antiparallel, and perpendicular magnetization with respect to the external field are present. For this configuration to be achieved, a domain rotation from the exter-
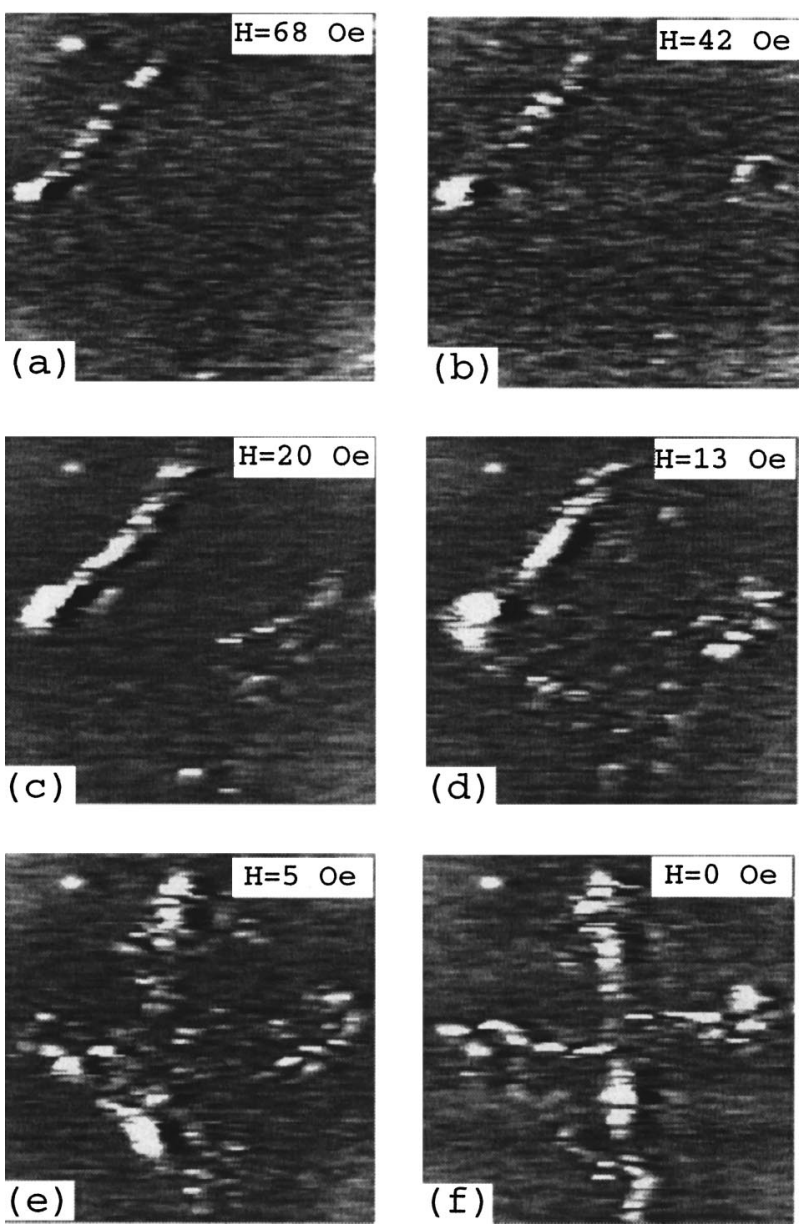

FIG. 4. Evolution of the dissipation configuration with decreasing magnetic field after saturation along one edge of the permalloy square. (a)-(f) are simultaneously acquired with (a)-(f) of Fig. 3, respectively.

nal field direction to a direction perpendicular to the field (parallel to the edge) must have occurred when the long magnetic structure meets other domain walls. The exact field at which this happens could not be determined. Possibly, this might be due to the limited time resolution of our nonoptimized imaging (each image takes $10-20 \mathrm{~min}$ ). The rotated domain is indicated in Fig. 4(e) by an arrow. Inside this rotated domain, another long magnetic structure is seen, which disappears when the external field is reduced to zero. At this point, the four domains become equal in size and the edge effect driven closure domain configuration is formed. The magnetic domain structure in Fig. 4(f) differs from the ac demagnetized sample in that the magnetic ripples appear only on two edges of the sample instead of on all the four edges. ${ }^{1}$ The magnetization direction of the two domains, which shows magnetic ripples, is either parallel or antiparallel to the previously applied field.

The magnetic dissipation signals (Fig. 4) show maxima always associated with domain-wall positions during the reversal.

Applying an external magnetic field does not move some structures [e.g., the "crater" in the middle of the square and the bright spots outside the square in Fig. 4(a)]. This indicates that they are topography that was not served out and 

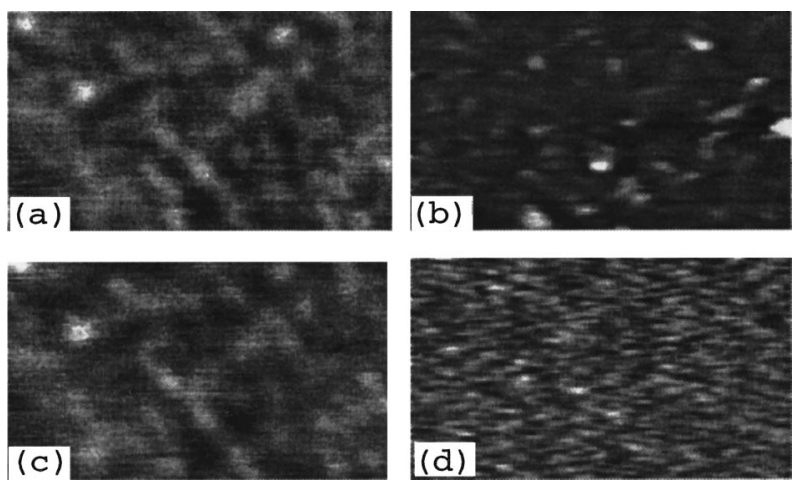

FIG. 5. Constant force gradient and magnetic dissipation images of an $\mathrm{Fe} / \mathrm{Cu}$ multilayer (GMR) sample. Image size is $5 \mu \mathrm{m}$ by $2.9 \mu \mathrm{m}$. The average tip-sample separation is $60 \mathrm{~nm}$. (a) and (b) are the constant force gradient and the simultaneously acquired dissipation images in the absence of an external field. (c) and (d) are the constant force gradient and the simultaneously acquired dissipation images in the presence of an external field of $30 \mathrm{Oe}$ on the same area as (a). Maximum $z$ variations in both (a) and (c) are all $1 \mathrm{~nm}$. The variation of driving amplitude in (b) is $70 \mathrm{mV}$ (corresponding to an energy loss of $0.019 \mathrm{eV}$ in one oscillation cycle, $Q_{0}$ $=10000$ for this experiment), while in (d) it is $18 \mathrm{mV}$ (thermal noise level). The average driving amplitude for (b) and (d) is $3000 \mathrm{mV}$. No image processing except for a plane subtraction was performed for the constant force gradient images. The dissipation images are raw data.

provided convenient markers that were independent of the magnetic structures.

In multilayer GMR samples (consisting of alternative nonmagnetic and magnetic layers), the adjacent magnetic layers are antiferromagnetically coupled through a Ruderman-Kittel-Kasuya-Yosida interaction. ${ }^{13}$ A single nonmagnetic or magnetic layer has a typical thickness of only several angstroms. As a result, the sample stray field at positions thousands of angstroms above the sample surface is virtually zero since the fields from the adjacent magnetic layers cancel each other. This raises a challenge to image the magnetic domain structure in these samples by normal magnetic force microscopy, which relies on the interaction between the tip magnetization and sample stray field. Figures 5(a) and (b) show the experimental results on a $(\mathrm{Cu} 25 \AA / \mathrm{Fe} 11 \AA) \times 36$ multilayer GMR sample at $z$ $=60 \mathrm{~nm}$. The contrast in Fig. 5(a) comes from the sample surface topography while the contrast in Fig. 5(b) is a result of tip stray field influencing the sample magnetic structure. We verify this by imaging with an external field. At a field of 30 Oe parallel to the sample surface (the sample's coercivity is $3 \mathrm{Oe}$ ), the contrast in the dissipation image [Fig. 5(d)] disappears while the constant force gradient image [Fig. $5(\mathrm{c})$ ] is unchanged. The signal observed in Fig. 5(d) corresponds quantitatively to the thermal noise limited dissipation measurements. Magnetic dissipation microscopy can thus detect magnetic structures not observable by magnetic force microscopy. This might have future applications in investigating antiferromagnets, spin glasses, and patterned magnetic media as well as in high-density storage if data rates and reliability issues can be solved.
Further studies on the origin of the dissipation in the GMR samples might help to determine the interlayer coupling strength and micromagnetic structure in these samples.

In conclusion, the nonconservative interaction of the tip stray field with micromagnetic structures can be deduced by measuring dissipation in an ac MFM. We find that the major contribution to magnetic dissipation originates in magnetoelastic losses as a result of the tip-field coupling to the sample magnetization. Dissipation measurements are thermally limited. We have achieved a sensitivity of better than $2.0 \times 10^{-4} \mathrm{eV} / \sqrt{\mathrm{Hz}}$ per oscillation cycle of the tip in a vacuum MFM. Lateral resolution in dissipation imaging is at least as good as standard MFM imaging. Quantitative dissipation measurements in combination with micromagnetic simulations should allow an upper boundary to be put on the influence of the tip stray field on the switching behaviors of small magnetic particles. Dissipation measurements allow us to determine the onset of tip influences on the micromagnetic structures long before they are observable in regular MFM. Dissipation also allows us to distinguish between different micromagnetic wall structures such as Néel and Bloch walls, Bloch lines, etc. Furthermore, the presence of domain walls can be deduced with dissipation measurements when standard MFM fails, such as at the edges of permalloy samples (large topography variations) or when the sample stray field is very weak (e.g., in antiferromagnetically coupled multilayer thin-film structures). Quantitative dissipation imaging on a suitable reference sample can also be used as a relative calibration of tip stray fields, an important parameter in qualitative and quantitative interpretation of MFM data.

The authors acknowledge initial help with the PLL electronic by U. Dürig (IBM Research Division) and F. Battiston. The GMR sample was kindly provided by Dok Won Lee (McGill). We appreciate D. Ryan's critical comments on the manuscript. This work was supported by grants from the National Science and Engineering Research Council of Canada and Le Fonds pour la Formation des Chercheurs et l'Aide à la Recherche de la Province de Québec.

\footnotetext{
${ }^{1}$ P. Grütter, Y. Liu, P. LeBlanc, and U. Dürig, Appl. Phys. Lett. 71, 279 (1997).

${ }^{2}$ Y. Liu, B. Ellman, and P. Grütter, Appl. Phys. Lett. 71, 1418 (1997).

${ }^{3}$ Y. Liu, Ph.D. thesis, McGill University, 1997.

${ }^{4}$ Y. Liu and P. Grütter, J. Appl. Phys. 83, 5922 (1998).

${ }^{5}$ W. Denk and D. W. Pohl, Appl. Phys. Lett. 59, 2171 (1991).

${ }^{6}$ U. Dürig, H. R. Steinauer, and N. Blanc, J. Appl. Phys. 82, 3641 (1997).

${ }^{7}$ G. Lévêque, P. Girard, S. Belaidi, and G. Cohen Solal, Rev. Sci. Instrum. 68, 4137 (1997).

${ }^{8}$ D. Rugar, H. L. Mamin, P. Guethner, S. E. Lambert, J. E. Stern, I. McFadyen, and T. Yogi, J. Appl. Phys. 68, 1169 (1990).

${ }^{9}$ H. J. Williams, W. Shockley, and C. Kittle, Phys. Rev. 80, 1090 (1950).

${ }^{10}$ H. W. Fuller and M. E. Hale, J. Appl. Phys. 31, 238 (1960).

${ }^{11}$ H. J. Mamin, D. Rugar, J. E. Stern, R. E. Fontana, Jr., and P. Kasiraj, Appl. Phys. Lett. 55, 318 (1989).

${ }^{12}$ P. LeBlanc, Master thesis, McGill University, 1996.

${ }^{13}$ P. Bruno and C. Chappert, Phys. Rev. Lett. 67, 1602 (1991).
} 